\title{
Variability and effects of bronchial colonisation in patients with moderate
} COPD

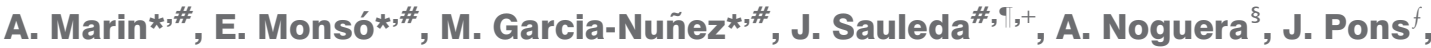 \\ A. Agusti ${ }^{\prime, q_{* * *}}$ and J. Morera*,\#
}

ABSTRACT: Sputum and lung function were periodically assessed in stable moderate chronic obstructive pulmonary disease (COPD) outpatients to determine relationships between bronchial colonisation and inflammation.

Relationships between potentially pathogenic microorganism (PPM) typology, bronchial inflammation (neutrophilia, tumour necrosis factor- $\alpha$, interleukin (IL)-1及, IL-6, IL-8, IL-10 and IL12) and post-bronchodilator decline in forced expiratory volume in $1 \mathrm{~s}$ (FEV1) were analysed. PPMs periodically showing the same molecular profile using pulse field gel electrophoresis were considered long-term persistent.

Bronchial colonisation was observed in 56 out of 79 follow-up examinations (70.9\%) and was mainly due to Haemophilus influenzae, Pseudomonas aeruginosa and enterobacteria $(n=47)$. These PPMs were all related to sputum neutrophilia $(\mathrm{p} \leqslant \mathbf{0 . 0 5}$, Chi-squared test), and $\boldsymbol{H}$. influenzae was related to higher levels of IL-1 $(p=0.005)$ and IL-12 $(p=0.01)$, with a dose-response relationship (Spearman's correlation coefficient of 0.38 for IL-1 $(p=0.001)$, and of 0.32 for IL-12 $(p=0.006))$. Haemophilus parainfluenzae was not associated with an identifiable inflammatory response. Long-term persistence of the same strain was observed in 12 examinations (21.4\%), mainly due to $P$. aeruginosa or enterobacteria. A neutrophilic bronchial inflammatory response was associated with a statistically significant decline in $\mathrm{FEV} 1$ during follow-up (OR $2.67,95 \% \mathrm{CI}$ 1.07-6.62).

A load-related relationship to bronchial inflammation in moderate COPD was observed for colonisation by $\boldsymbol{H}$. influenzae, but not for colonisation by $\boldsymbol{H}$. parainfluenzae.

KEYWORDS: Bronchial colonisation, chronic obstructive pulmonary disease, Haemophilus influenzae, interleukin-1 $\beta$, interleukin-12, potentially pathogenic microorganisms

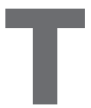

he bronchial tree and the pulmonary parenchyma are sterile in healthy subjects, but in patients with chronic obstructive pulmonary disease (COPD) potentially pathogenic microorganisms (PPMs) are often recovered from bronchial secretions during periods of clinical stability and, particularly, during episodes of exacerbation, when PPM loads increase significantly $[1,2]$. Most studies on PPM colonisation in COPD have focused on patients with severe disease [3-5]. In contrast, the relationships between the microbiology of bronchial colonisation and local inflammatory response in moderate COPD patients, when therapeutic interventions are expected to have maximal effects, have only been assessed in small selected population samples [6-8]. With the hypothesis that the characteristics of bronchial colonisation have an effect on bronchial inflammation and lung function that may be identified in stable outpatients with moderate COPD, the present study sought: first to determine the prevalence and load of bronchial colonisation in a cohort of COPD outpatients with moderate airflow limitation never admitted to a hospital for an exacerbation of the disease; and secondly, to investigate in these patients the relationships between colonisation characteristics, bronchial inflammation markers and post-bronchodilator changes in forced expiratory volume in 1 s (FEV1) during follow-up.

\section{METHODS}

Design and study population

We enrolled a cohort of stable COPD outpatients diagnosed according to the criteria of the Global Initiative for Chronic Obstructive Lung Disease (GOLD) [9] and reporting no admissions for respiratory symptoms. Patients were examined at

\section{AFFILIATIONS}

${ }^{*}$ Respiratory Medicine Dept, Hospital Germans Trias I Pujol, Badalona, Universidad Autonoma de Barcelona

"Respiratory Medicine Dept,

${ }^{\text {s}}$ Clinical Analysis Dept and,

fImmunology Dept, Hospital Son

Dureta, Palma de Mallorca,

**Hospital Clínic i Provincial,

Barcelona,

+Fundació Caubet-CIMERA, and

\# Ciber de Enfermedades

Respiratorias - CIBERES, Bunyola, Spain.

CORRESPONDENCE

E. Monsó

Servei de Pneumologia

Hospital Germans Trias i Pujol

Carretera de Canyet $s / n$

08916 Badalona

Catalonia

Spain

E-mail: emonso.germanstrias@

gencat.cat

Received:

Aug 182008

Accepted after revision:

July 152009

First published online:

July 302009

European Respiratory Journal

Print ISSN 0903-1936

Online ISSN 1399-3003 
baseline in stable condition from $\geqslant 8$ weeks, and their sociodemographic data, smoking habits, respiratory symptoms, treatments, sputum microbiological and inflammatory characteristics, and lung function were recorded. 9 months (interval 7-11 months) after this baseline assessment, a follow-up visit was scheduled at a time when the patient had been in a stable condition for $\geqslant 8$ weeks. That visit included a second assessment of sputum characteristics, together with recording of exacerbation history and assessment of lung function changes since the previous visit. Patients were excluded if they: were $<40$ yrs of age; had ever been admitted to a hospital for respiratory symptoms; were diagnosed with asthma, cystic fibrosis, neoplasia or bronchiectasis, and/or were being treated with oral corticosteroids or immunosuppressors for any reason. Additionally, patients who did not maintain the same smoking habits and baseline bronchodilator and inhaled corticosteroid treatment throughout the follow-up interval were excluded from analysis. Acute episodes of increased dyspnoea, sputum production and/or purulence appearing during follow-up and treated with antibiotics and/or oral corticosteroids by a physician were considered as exacerbations $[10,11]$. The present study was reviewed and approved by the local research ethics committee in Catalonia, Spain.

\section{Interview questionnaire and lung function}

The interview questionnaire, which included items on age, sex and chronic bronchitis (defined as cough and phlegm $>3$ months each yr) was obtained from the European Community Respiratory Health Survey (ECRHS) [12, 13]. Current treatments and previous exacerbations were also recorded. All subjects performed forced spirometry and reversibility tests in the morning with the same dry rollingseal spirometer (Spirometrics, Gray, ME, USA) at baseline and follow-up visits according to standard techniques [14]. Forced vital capacity and FEV1 were measured and were compared with age- and height-adjusted reference values obtained from selected volunteers from the Barcelona province [15]. This was followed by a reversibility test with salbutamol. Results were expressed as absolute values (mL) and percentages of the reference values.

\section{Sputum sampling and analysis}

An induced sputum sample was obtained and processed within $60 \mathrm{~min}$ at each visit according to standard methods [16, 17]. In brief, the patient was pre-treated with an inhaled $\beta_{2^{-}}$ agonist $10 \mathrm{~min}$ before nebulisation of isotonic saline $(0.9 \%)$; this was followed by increasing concentrations of hypertonic saline $(3,4$ and $5 \%$ ) for $7 \mathrm{~min}$ with each concentration. After each induction the patient attempted to obtain a sputum sample by coughing, and the nebulisation procedure was halted when the sputum volume collected was $\geqslant 1 \mathrm{~mL}$ [18]. Sputum induction was performed after $6 \mathrm{~h}$ of abstinence in current smokers. Samples with $>25$ leukocytes per field (Murray-Washington $\geqslant 3$ ) were considered indicative of a neutrophilic inflammatory response [19, 20].

\section{Microbiological determinants}

The sputum sample was weighed and processed with an equal volume of dithiothreitol (Sputasol; Oxoid Ltd, Basingstoke, UK), cultured, and the microbial load of the sample was determined [21]. The determination of microbial typology and load was carried out by means of serial dilutions and culture in selective media, according to standard methods [22], with quantitative cultures expressed as colony-forming units $(\mathrm{cfu}) \cdot \mathrm{mL}^{-1}$. For the purposes of this study, cultures were considered positive for bronchial colonisation according to previously defined criteria [23-25] if they grew microorganisms, at loads of $\geqslant 100 \mathrm{cfu} \cdot \mathrm{mL}^{-1}$, that were considered as potentially pathogenic, such as Haemophilus influenzae, Haemophilus parainfluenzae, Streptococcus pneumoniae, Moraxella catarrhalis, Pseudomonas aeruginosa, enterobacteria and/or Staphylococcus aureus.

\section{Molecular genotyping}

Molecular PPM typing was performed using pulse field gel electrophoresis (PFGE) to determine whether a PPM recovered from consecutive samples corresponded to the persistence of the same strain or to the acquisition of a new one [26]. In brief, chromosomal DNA from multiple (more than four) PPMs growing in chocolate agar was extracted with agarose and incubated at $37^{\circ} \mathrm{C}$ for $18 \mathrm{~h}$. After enzyme digestion with Sma I (New England Biolabs, Ipswich, MA, USA), restriction fragments were separated in agarose gel with tris-borate-ethylenediaminetetraacetic acid (Sigma Chemical, St Louis, MI, USA) through PFGE using a homogeneous electric camp contour clamp (CHEF DR II system; BioRad, Ivey sur Seine, France), beginning with an initial 5.6-s pulse that was linearly increased until a 40.6-s pulse was reached and then maintained for $24 \mathrm{~h}$ at $5 \mathrm{~V} \cdot \mathrm{cm}^{-1}$ and $14^{\circ} \mathrm{C}$. Bacteriophage $\lambda$ concatemer (New England Biolabs) was included as the molecular weight DNA marker. Obtained patterns were photographed with a 360-nm transilluminator after staining, and analysed using Diversity Database Software (BioRad). PPMs cultured from follow-up sputum samples obtained from patients who grew the same species at baseline and showed the same molecular profile in both samples analysed by PFGE were considered long-term persistent strains, whereas PPMs cultured at follow-up which were not present in the baseline sample were considered as strains acquired during follow-up (fig. 1).

\section{Inflammatory markers}

The remaining sputum was centrifuged and the concentrations of several cytokines (tumour necrosis factor (TNF)- $\alpha$, interleukin (IL)-1 $\beta$, IL-6, IL-8, IL-10 and IL-12) were measured in the supernatant using a cytokine bead array (BD Biosciences, San Diego, CA, USA). This assay system consists of a mixture of uniform bead types that contain different fluorescence intensities of a red-emitting dye. A capture antibody against each cytokine is covalently coupled to a type of bead, and cytokines bound to these antibodies are detected by use of phycoerythrin-labelled antibodies. The fluorescence intensity measured with phycoerythrin is proportional to the cytokine concentration in the sample and is quantified for every cytokine from a calibration curve. The detection limits of these assays were $3.7 \mathrm{pg} \cdot \mathrm{mL}^{-1}$ for TNF- $\alpha, 7.2 \mathrm{pg} \cdot \mathrm{mL}^{-1}$ for IL-1 $\beta$, $2.5 \mathrm{pg} \cdot \mathrm{mL}^{-1}$ for IL-6, $3.6 \mathrm{pg} \cdot \mathrm{mL}^{-1}$ for IL-8, $3.3 \mathrm{pg} \cdot \mathrm{mL}^{-1}$ for IL-10 and $1.8 \mathrm{pg} \cdot \mathrm{mL}^{-1}$ for IL-12.

\section{Statistical analysis}

All data were added to a database and analysed using the SPSS statistical software package version 15 (Chicago, IL, USA). Results are expressed as absolute and relative frequencies for 
a) Case 1

Change of genotype

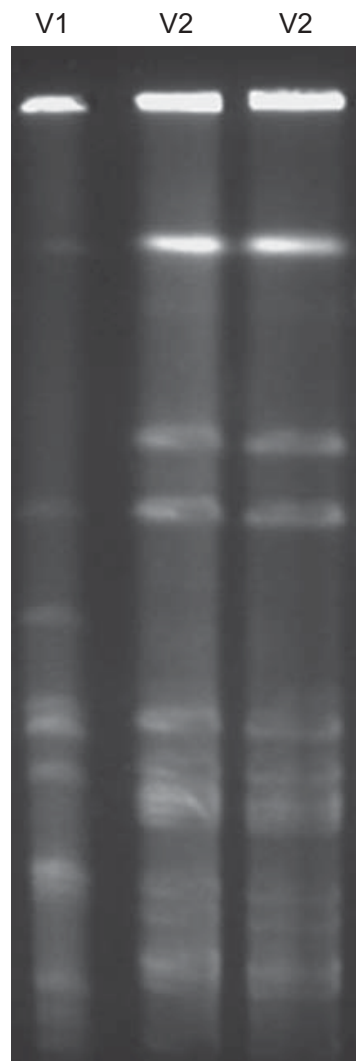

b) Case 2

Persistent genotype and acquired genotype

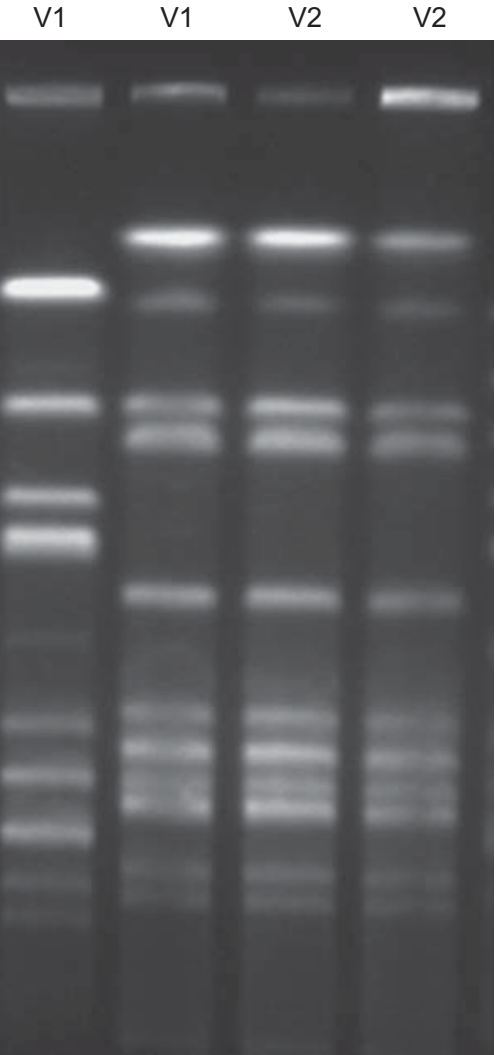

FIGURE 1. Pulse-field electrophoresis of samples growing Haemophilus influenzae in baseline and follow-up samples. a) Persistent $H$. influenzae strain, and b) appearance of a strain newly acquired during follow-up.

categoric variables and as means $\pm \mathrm{SD}$, or median (interquartile range (IQR)) for continuous variables, as appropriate depending on the distribution of data. A value of half the lower detection limit was used for all measures of continuous variables showing a result below that value.

Descriptive statistics were compiled for sociodemographic characteristics and all the examinations were performed at baseline and at the end of follow-up. Sputum characteristics recorded at both visits were microbial profile and load, neutrophilia according to Murray-Washington criteria $(\geqslant 3)$, and concentration of cytokines (TNF- $\alpha$, IL-1 $\beta$, IL- 6 , IL-8, IL-10 and IL-12). Additional follow-up measurements were length of time in months between visits, appearance and frequency of exacerbations (calculated as number of exacerbations in the period/number of months of follow-up $\times 12$ ); and change in the post-bronchodilator FEV1 in $\mathrm{mL}$ during the follow-up period (calculated as follow-up FEV1 - previous FEV1/number of months of follow-up $\times 12$ ).

Analysis of the relationships between microbial typology and bronchial inflammation, measured as sputum neutrophilia and cytokine concentration, were performed at the end of followup (Chi-squared, Fisher's exact and Mann-Whitney U-tests). In this analysis, PPMs cultured from follow-up sputum samples and showing the same molecular profile as PPMs recovered at

\section{TABLE 1 Sociodemographic characteristics}

\begin{tabular}{|c|c|}
\hline Patients n & 40 \\
\hline Age yrs & $66.5 \pm 8.1$ \\
\hline Males & $39(97.5)$ \\
\hline Ever-smokers & $40(100.0)$ \\
\hline Current & $8(20.0)$ \\
\hline Former & $32(80.0)$ \\
\hline Chronic bronchitis & $28(70.0)$ \\
\hline Exacerbations previous yr & $0(0-2)$ \\
\hline Use of inhaled steroids & $29(72.5)$ \\
\hline \multicolumn{2}{|l|}{ Post-bronchodilator } \\
\hline $\mathrm{FEV}_{1} \%$ & $57.9 \pm 19.1$ \\
\hline FVC \% & $89.4 \pm 23$ \\
\hline $\mathrm{FEV}_{1} / \mathrm{FVC} \%$ & $50.4 \pm 10.2$ \\
\hline $\mathrm{Pa}, \mathrm{O}_{2} \mathrm{mmHg}$ & $76.8(10.9)$ \\
\hline $\mathrm{Pa}, \mathrm{CO}_{2} \mathrm{mmHg}$ & 40.5 (3.5) \\
\hline
\end{tabular}

Data are presented as mean $\pm \mathrm{SD}, \mathrm{n}(\%)$, or median (interquartile range), unless otherwise stated. FEV1: forced expiratory volume in $1 \mathrm{~s}$; FVC: forced vita capacity; $\mathrm{Pa}_{\mathrm{a}} \mathrm{O}_{2}$ : arterial partial pressure of oxygen; $\mathrm{Pa}_{\mathrm{a}} \mathrm{CO}_{2}$ : arterial partia pressure of carbon dioxide.

baseline were considered as long-term persistent strains, whereas PPMs cultured at follow-up but not present at baseline were considered as acquired. First, correlations between microbial load and bronchial inflammation parameters (Mann-Whitney U-test and the Spearman's correlation coefficient) were calculated. Next, the differences between colonised patients who harboured long-term persistent PPMs and microorganisms acquired during follow-up were analysed, through the assessment of the relationships between long-term microbial persistence and microbial profile, load, sputum neutrophilia and concentration of cytokines at the follow-up visit. Finally, the impact of bronchial inflammation on the change in post-bronchodilator FEV1 during the followup period was assessed using logistic regression modelling, considering an FEV1 decline greater than the median decline as the outcome, and age, current smoking, use of inhaled corticosteroids and appearance of an exacerbation during follow-up as covariates. The results of these analyses were expressed as crude and adjusted OR with 95\% CI. Multivariate models were adjusted for smoking and other covariates showing an association with the outcome variable in the univariate models $(p<0.20)$. All statistical tests were two sided, and a $p$-value of $\geqslant 0.05$ less was reported as statistically significant.

\section{RESULTS}

40 consecutive, clinically stable COPD outpatients fulfilled the inclusion criteria and were enrolled. Participating patients were mostly male (39 $(97.5 \%))$, had a mean age $\pm S D$ of $66.5 \pm 8.1$ yrs and showed moderate lung function impairment (post-bronchodilator FEV1 $57.9 \%$ of predicted (SD 19.1\%)) (table 1). In these patients, 79 baseline visits and consecutive follow-up examinations were performed after $8.0 \pm 3.2$ months. Sputum was obtained with isotonic saline in 23 baseline and 34 follow-up visits, and was induced with hypertonic saline in the remaining examinations. PPMs were recovered from nearly three-quarters of these sputum samples, both at baseline and at 


\begin{tabular}{|c|c|c|c|}
\hline \multirow[t]{3}{*}{ TABLE 2} & ics of sputum & sample $(\mathrm{n}=7$ & \\
\hline & \multicolumn{3}{|c|}{ Sputum } \\
\hline & Baseline & Follow-up & $\mathrm{p}$-value \\
\hline PPMs & $58(73.4)$ & $56(70.9)$ & $>0.25$ \\
\hline Sputum neutrophilia" & $53(67.1)$ & $40(50.6)$ & $>0.25$ \\
\hline PPMs and neutrophilia ${ }^{\#}$ & $45(57.0)$ & $35(44.3)$ & 0.15 \\
\hline Polymicrobial & $10(12.7)$ & $9(11.4)$ & $>0.25$ \\
\hline \multicolumn{4}{|l|}{ Microbial typing } \\
\hline H. influenzae & $28(35.4)$ & $25(31.6)$ & $>0.25$ \\
\hline Load cfu $\cdot \mathrm{mL}^{-1} \times 10^{3}$ & $100(20-10000)$ & $700(10-4500)$ & $>0.25$ \\
\hline $\begin{array}{l}P . \text { aeruginosa or } \\
\text { enterobacteria }\end{array}$ & $18(22.8)$ & $22(27.8)$ & $>0.25$ \\
\hline Load cfu $\cdot \mathrm{mL}^{-1} \times 10^{3}$ & $2(1-10)$ & $1(1-6)$ & $>0.25$ \\
\hline H. parainfluenzae & $16(20.2)$ & $16(20.2)$ & $>0.25$ \\
\hline Load cfu $\cdot \mathrm{mL}^{-1} \times 10^{3}$ & $100(10-115)$ & $55(6-165)$ & $>0.25$ \\
\hline M. catarrhalis & $4(5.1)$ & $2(2.5)$ & $>0.25$ \\
\hline $\mathrm{TNF}-\alpha \mathbf{p g} \cdot \mathrm{mL}^{-1}$ & $9(5-29)$ & $18(4-41)$ & $>0.25$ \\
\hline $\mathrm{IL}-1 \beta \mathrm{pg} \cdot \mathrm{mL}^{-1}$ & $664(173-1619)$ & $837(179-2493)$ & $>0.25$ \\
\hline $\mathrm{IL}-6 \mathrm{pg} \cdot \mathrm{mL}^{-1}$ & $692(182-2694)$ & $494(120-1834)$ & $>0.25$ \\
\hline $\mathrm{IL}-8 \mathrm{ng} \cdot \mathrm{mL}^{-1}$ & $31(8-44)$ & $40(23-65)$ & 0.02 \\
\hline $\mathrm{IL}-10 \mathrm{pg} \cdot \mathrm{mL}^{-1}$ & $6(4-13)$ & $10(3-27)$ & 0.16 \\
\hline $\mathrm{IL}-12 \mathrm{pg} \cdot \mathrm{mL}^{-1}$ & $7(1-17)$ & $7(1-23)$ & $>0.25$ \\
\hline
\end{tabular}

Data are present as $\mathrm{n}(\%)$ and median (interquartile range). PPM: potentially pathogenic microorganisms; $H$. influenzae: Haemophilus influenzae; $P$. aeruginosa: Pseudomonas aeruginosa; $H$. parainfluenzae: Hemophilus parainfluenzae; M. catarrhalis: Moraxella catarrhalis; cfu: colony-forming unit; TNF: tumour necrosis factor; IL: interleukin. ${ }^{*}$ : Murray-Washington score $\geqslant 3$.

the follow-up visits, and in half of them this recovery was associated with a neutrophilic response. $H$. influenzae was the PPM recovered most often (in 28 and 25 cases at baseline and follow-up visits, respectively), and samples growing that microorganism also had the highest microbial loads. Low loads of $P$. aeruginosa, enterobacteria and $H$. parainfluenzae, however, were also often cultivated from the sputum samples (table 2).

59 samples showed bronchial colonisation at the follow-up examination, preceded by positive sputum cultures for PPMs in the baseline sputum sample in $42(53.2 \%)$ cases. These successive positive cultures, however, were in most cases due to new PPMs acquired during follow-up (30 (38.0\%) cases). Long-term persistence of the same strain was the cause in only $12(15.2 \%)$ cases, a situation that was mainly attributable to colonisation by $P$. aeruginosa or enterobacteria (table 3 ). No statistically significant differences in the measured bronchial inflammation parameters were found between sputum samples with long-term persistent and acquired colonisation (table 4).

The identification of bronchial colonisation by H. influenzae in the follow-up sputum was associated with a bronchial inflammatory response identifiable through a higher prevalence of sputum neutrophilia in samples positive for this PPM (68.0 versus $42.6 \% ; \mathrm{p}=0.04$, Chi-squared test), and higher concentrations of IL-1 $\beta$ (median 1,636 (IQR 597-7,736) versus
TABLE 3 Evolution during follow-up $(n=79)$

\begin{tabular}{|c|c|}
\hline Months follow-up & $8.0 \pm 3.2$ \\
\hline$\geqslant 1$ exacerbation during follow-up & $21(26.6)$ \\
\hline Exacerbations in follow-up per yr & $0(0-1)$ \\
\hline Change FEV1 post-bronchodilation $\mathrm{mL} \cdot \mathrm{yr}^{-1}$ & $-72(-270-120)$ \\
\hline Former smokers $\#$ & $-50(-390-126)$ \\
\hline Current smokers $\#$ & $-76(-240-90)$ \\
\hline \multicolumn{2}{|l|}{ Sputum cultures baseline and follow-up } \\
\hline PPMs in both & $42(53.2)$ \\
\hline Same PPM strain in both & $12(15.2)$ \\
\hline PPMs and neutrophilia in both & $16(20.3)$ \\
\hline Same PPM strain and neutrophilia in both & $12(15.2)$ \\
\hline
\end{tabular}

Data are presented as mean $\pm \mathrm{SD}, \mathrm{n}(\%)$ or median (interquartile range). FEV1: forced expiratory volume in $1 \mathrm{~s}$; PPM: potentially pathogenic microorganisms. \#: difference statistically nonsignificant ( $p>0.25$, Mann-Whitney U-test)

$\because$ : Murray-Washington score $\geqslant 3$

601 (153-1,320) $\mathrm{pg} \cdot \mathrm{mL}^{-1} ; \mathrm{p}=0.005$, Mann-Whitney U-test) and IL-12 (14 (7-29) versus 2 (1-17) $\mathrm{pg} \cdot \mathrm{mL}^{-1} ; \mathrm{p}=0.01$, MannWhitney U-test) (fig. 2; table 5). This inflammatory response was not observed in samples colonised by $P$. aeruginosa/ enterobacteria or $H$. parainfluenzae (table 5). The recovered load of $H$. influenzae was also positively correlated with the sputum concentrations of IL-1 $\beta$ in patients with sputum cultures positive for this PPM ( $\rho=0.64, p=0.001$, Spearman's correlation coefficient) (fig. 3). In samples colonised by $P$. aeruginosa or enterobacteria, a neutrophilic inflammatory response was also observed ( $p=0.05$, Chi-squared test) (table 5). This neutrophilic inflammatory response, mainly found in patients colonised by H. influenzae, P. aeruginosa or enterobacteria (27 out of 40 neutrophilic samples, $67.5 \%$ ), showed a statistically significant relationship with FEV1 declines over the median decline during follow-up in our sample of moderate COPD patients (OR 2.67, 95\% CI 1.07-6.62; $\mathrm{p}=0.03$ ). That relationship was not modified after adjustment for covariates and was not found for IL-1 $\beta$ or IL-12, nor were statistically significant relationships found between inhaled corticosteroid use or appearance of an exacerbation and follow-up inflammatory markers in sputum (data not shown).

\section{DISCUSSION}

In the present study, focusing on the effects of bronchial colonisation on inflammation and lung function in moderate COPD, we found that PPMs were recovered from bronchial secretions in nearly three-quarters of the patients. The recovery of $H$. influenzae, P. aeruginosa and enterobacteria from bronchial secretions was closely related to a neutrophilic response, suggesting that the cellular inflammatory effects of bronchial colonisation on moderate COPD depend mainly on the presence of these PPMs. $H$. influenzae colonisation was additionally associated with higher sputum concentrations of such inflammation markers as IL-1 $\beta$ and IL-12, a relationship that was shown to be load-mediated for IL-1 $\beta$. Bronchial colonisation by $H$. parainfluenzae, however, was not associated with an inflammatory response, a finding suggesting that the effect of this microorganism on the bronchial mucosa must be considered as marginal. The repetition of the sputum cultures was recurrently positive for PPMs in more than half of the 


\begin{tabular}{|c|c|c|c|}
\hline \multirow[t]{3}{*}{ TABLE 4} & $\begin{array}{l}\text { bial load and in } \\
\text { up samples sh } \\
\text { ding to persiste } \\
\text { genic microorg }\end{array}$ & $\begin{array}{l}\text { lammatory ma } \\
\text { owing colonisa } \\
\text { hce of potentia } \\
\text { inisms }\end{array}$ & $\begin{array}{l}\text { rs in } \\
n\end{array}$ \\
\hline & \multicolumn{3}{|c|}{ PPM strain } \\
\hline & Persistent & Acquired & p-value \\
\hline Samples n & 12 & 44 & \\
\hline H. influenzae & $5(41.7)$ & $20(42.5)$ & $>0.25$ \\
\hline Load $^{\#} \mathrm{cfu} \cdot \mathrm{mL}^{-1} \times 10^{3}$ & $3000(50-10000)$ & $550(10-2750)$ & 0.23 \\
\hline $\begin{array}{r}\text { P. aeruginosa or } \\
\text { enterobacteria }\end{array}$ & $9(75.0)$ & $13(27.7)$ & 0.006 \\
\hline Load $^{\#} \mathrm{cfu} \cdot \mathrm{mL}^{-1} \times 10^{3}$ & $1(1-10)$ & $1(1-3)$ & $>0.25$ \\
\hline H. parainfluenzae & $2(16.7)$ & $14(29.8)$ & $>0.25$ \\
\hline Load $^{\#} \mathrm{cfu} \cdot \mathrm{mL} \mathrm{L}^{-1} \times 10^{3}$ & $10,60^{\circ}$ & $55(6-165)$ & $>0.25$ \\
\hline \multicolumn{4}{|l|}{ Inflammatory markers } \\
\hline Sputum neutrophilia $^{+}$ & $8(66.7)$ & $27(61.4)$ & $>0.25$ \\
\hline $\mathrm{TNF}-\alpha \mathrm{pg} \cdot \mathrm{mL}^{-1}$ & $21(9-56)$ & $19(3-48)$ & $>0.25$ \\
\hline $\mathrm{IL}-1 \beta \mathrm{pg} \cdot \mathrm{mL}^{-1}$ & $1404(662-2790)$ & $1095(188-5323)$ & $>0.25$ \\
\hline $\mathrm{IL}-6 \mathrm{pg} \cdot \mathrm{mL}^{-1}$ & $664(156-2335)$ & 557 (151-2532) & $>0.25$ \\
\hline $\mathrm{IL}-8 \mathrm{ng} \cdot \mathrm{mL}^{-1}$ & $42(15-55)$ & 41 (24-92) & $>0.25$ \\
\hline $\mid \mathrm{L}-10 \mathrm{pg} \cdot \mathrm{ml}^{-1}$ & $14(6-31)$ & $10(3-29)$ & $>0.25$ \\
\hline $\mathrm{IL}-12 \mathrm{pg} \cdot \mathrm{mL}^{-1}$ & $16(9-26)$ & $7(1-21)$ & 0.08 \\
\hline \multicolumn{4}{|c|}{ 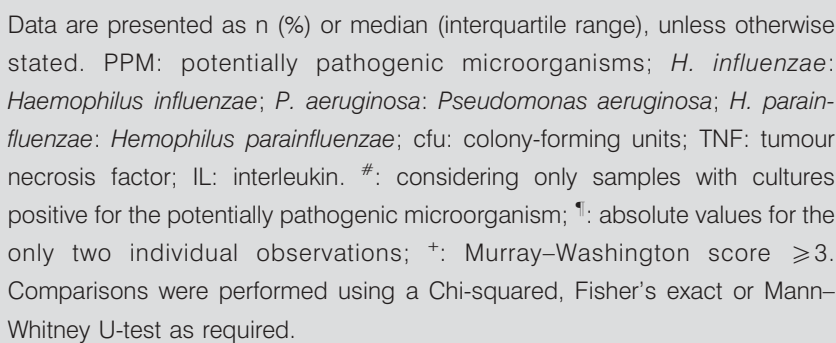 } \\
\hline
\end{tabular}

patients, in most cases due to colonisation during follow-up by a newly acquired PPM; thus, long-term persistence of the same strain was observed in fewer than a fifth of the cases, all, however, with an associated neutrophilic response. The finding of sputum neutrophilia during stability was associated with a significant lung function decline in these moderate COPD patients, a relationship that was independent of current smoking.

Previous studies of bronchial colonisation in stable COPD patients, in which the protected specimen brush was used to collect microbiology samples, have demonstrated cultures positive for PPMs in one-third of the patients, with $H$. influenzae, S. pneumoniae and $M$. catarrhalis the PPMs most often recovered [1]. When sputum has been used for the identification of bronchial colonisation in COPD, the cultures positive for PPMs have been found in one-fifth [5] to threequarters [4] of the samples. In patients unable to spontaneously produce appropriate sputum samples during their stable periods, the use of sputum induction has facilitated the study of bronchial colonisation by allowing most stable COPD patients to be sampled [6, 27]. Study of the cytological characteristics of sputum in this context has shown cultures positive for PPMs with neutrophilia in half of the studied cases
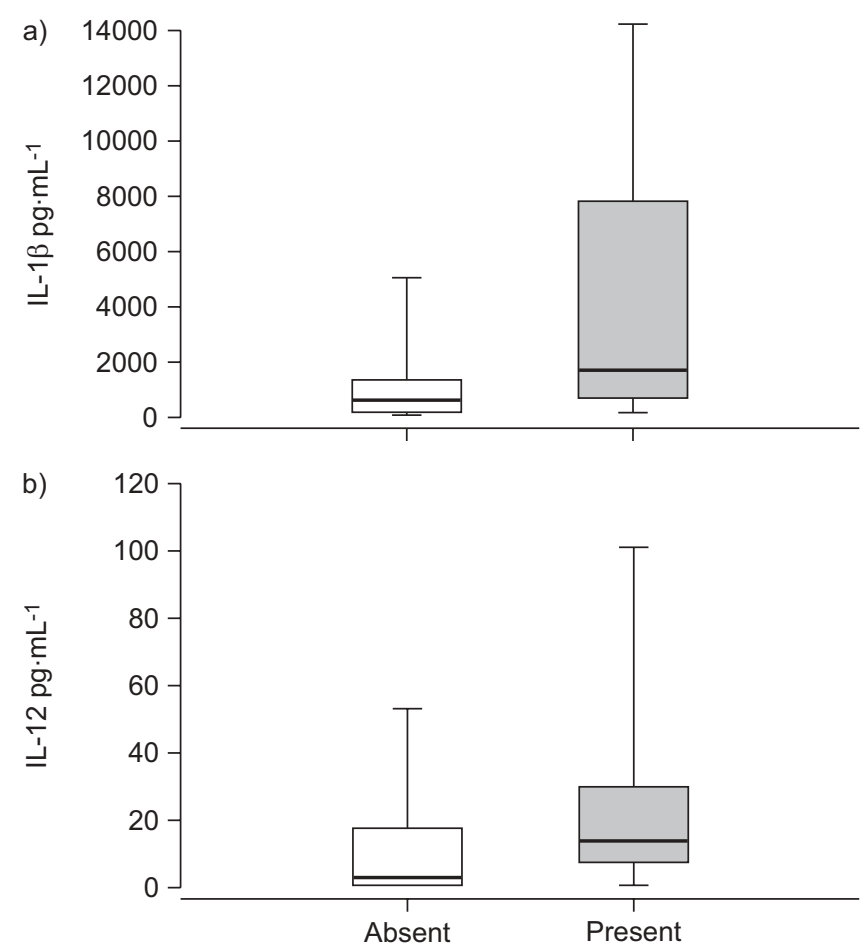

FIGURE 2. a) Interleukin (IL)-1 $\beta$ and b) IL-12 inflammatory markers in sputum according to the presence of bronchial colonisation by Haemophilus influenzae. Boxes represent median (horizontal dividing line) and interquartile range; whiskers represent SD.

$[3,28]$, a rate similar to the prevalence found in the present study, in which $H$. influenzae was the most frequently isolated PPM.

Bronchial colonisation by $H$. influenzae was significantly related to sputum neutrophilia and higher sputum concentrations of the inflammatory mediators IL- $1 \beta$ and IL- 12 in our sample of stable COPD patients with moderate lung function impairment. These observations suggest that colonisation by these PPMs is already able to induce an inflammatory response in the bronchial mucosa of such patients. This inflammatory response was seen to be species-specific because in our series, a similar effect was not found in COPD patients colonised by $H$. parainfluenzae, a PPM that occasionally causes COPD exacerbation and pneumonia [29-31], but has been shown to exhibit low mucosal adherence and a minimal potential to cause bronchial inflammation [32, 33]. Our choice of $H$. influenzae load as a main study variable was justified by its close relationship to the inflammatory response of the bronchial mucosa. Most PPMs recovered from participating COPD patients grew low microbial loads $\left(\leqslant 10^{5} \mathrm{cfu} \cdot \mathrm{mL}^{-1}\right)$, with high microbial loads being found almost exclusively when $H$. influenzae was the colonising PPM in our study. High-load $H$. influenzae colonisation was significantly associated with neutrophilic sputum samples (Murray-Washington $\geqslant 3$ ) and higher levels of IL-1 $\beta$ in sputum. When dose-response relationships between PPM load and bronchial inflammatory mediators in sputum have been assessed in previous studies not focusing on $H$. influenzae, they have supported a causal role for bronchial colonisation in the pathogenesis of bronchial 


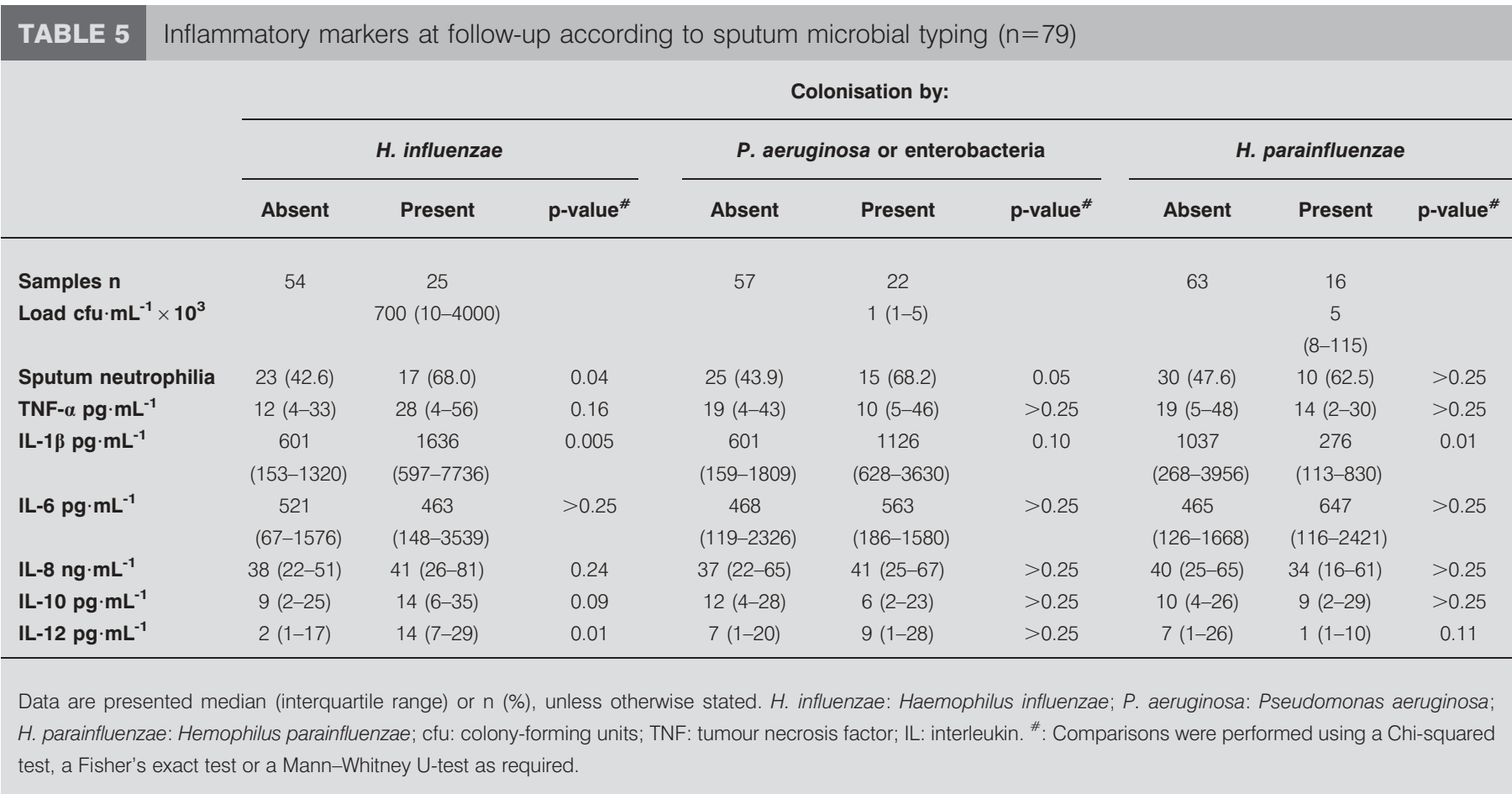

inflammation in COPD $[4,34,35]$, and loads $>10^{5} \mathrm{cfu} \cdot \mathrm{mL}^{-1}$ have usually been associated with neutrophilic inflammation [3]. Most series of patients with advanced disease, however, have shown positive correlations between microbial load and IL-8, myeloperoxidase, neutrophil elastase and leukotriene $B_{4}$ $[3,4,28]$. Together, all of these findings point to a significant impact of bronchial colonisation load on the mucosal inflammatory response, which, according to our results, may already be found, though with a different pattern, in patients with moderate disease when $H$. influenzae is the colonising bacteria. IL-1 $\beta$ has been related to bronchial colonisation in animal models [36], and our observation of high concentrations of this



FIGURE 3. Scatterplot of total bacterial count (colony-forming units (cfu) $\cdot \mathrm{mL}^{-1}$ ) and sputum interleukin (IL)-1 $1 \beta$ (Spearman's correlation coefficient $\rho=0.64$, $p=0.001$ ) in patients colonised by Haemophilus influenzae at the follow-up examination $(n=25)$. Bacterial count data have been logarithmically transformed. inflammatory mediator and IL-12, a cytokine related to the mucosal response against bacteria [37], in moderate COPD patients with $H$. influenzae colonisation supports the hypothesis of differences in the inflammatory response to bronchial colonisation according to the severity of lung function impairment. Our finding of a different pattern may have clinical implications and requires further assessment.

We have found that samples showing bronchial colonisation were preceded by positive sputum cultures for PPMs in more than half the cases, and we have used molecular typing techniques to investigate whether recovered PPMs corresponded to newly acquired or long-term persistent strains. According to this analysis, bronchial colonisation was mostly attributable to new PPMs rather than to persistent ones. Persistent colonisation was observed in fewer than a fifth of the samples that were positive on follow-up and was most often attributable to long-term colonisation by $P$. aeruginosa or enterobacteria. Two previous studies reported that microbial persistence accounted for about a half of the cases of bronchial colonisation in stable COPD patients [3,5], a prevalence higher than we have found. These studies, however, mostly investigated patients with severe COPD, and only one of them used molecular typing techniques to rule out colonisation by a new strain of the same PPM [5]. Our observations suggest that, in spite of a high prevalence of bronchial colonisation in moderate COPD patients, long-term microbial persistence is less often the cause, probably due to a higher turnover of colonising strains in less advanced disease.

Our findings in moderate COPD patients colonised by $H$. influenzae, $P$. aeruginosa and enterobacteria confirm the close association between colonisation by these PPMs and neutrophilia reported in other studies [34]. This association supports 
an early relationship between colonisation and the appearance of cellular inflammatory mediators in bronchial secretions, years before the disease causes severe impairment and at a stage when these relationships could be more easily identifiable $[38,39]$. The importance of the neutrophilic inflammatory response associated with bronchial colonisation is emphasised by our finding of a statistically significant relationship between the appearance of this inflammatory response and lung function impairment during follow-up. A similar functional impairment associated with bronchial colonisation has been documented by WILKINSON et al. [3], but was not found by HILL et al. [4]. In our study, the decline in FEV1 in COPD patients with sputum neutrophilia in some cases approached the magnitude previously seen in exacerbated COPD patients with more severe disease [40]. This finding supports the hypothesis that bronchial colonisation may have a subclinical impact in moderate COPD patients, who may be less prone to report their daily symptoms as an exacerbation [41]. Such patients may have functional impairment equivalent to the FEV1 decline that is associated with exacerbation in patients with advanced disease, who suffer easily from dyspnoea with even slight impairments in their lung function.

We have not performed genomic analysis of the sputum samples that do not grow PPMs, and we cannot rule out underdiagnosis of microbial persistence due to bronchial colonisation at loads below the detection limit of the sputum culture. This situation must be considered as unusual, however, because when this approach has been used undiagnosed, bronchial colonisation has only been identified in onetenth of the culture-negative sputum samples [42]. The lack of virological data is also a limitation of our study, but respiratory viruses are seldom recovered from bronchial secretions in stable COPD patients, who only give positive samples in onetenth of the cases $[43,44]$. The lowest prevalence of viral recovery has been found in infrequent exacerbators [44], such as those in our study.

In summary, we found that the bronchial tree of clinically stable outpatients with moderate COPD and without previous admissions due to respiratory diseases is often colonised by PPMs, but high sputum loads are only reached by $H$. influenzae. The presence of this PPM is associated with an inflammatory response of the bronchial mucosa, characterised by neutrophilic inflammation and high levels of IL-1 $\beta$ and IL-12, findings that are not characteristic of patients colonised by $H$. parainfluenzae. Colonising $P$. aeruginosa and enterobacteria are more often persistent and also associated with sputum neutrophilia. These observations suggest that the effect of bronchial colonisation on COPD patients is mainly mediated by $H$. influenzae, $P$. aeruginosa and enterobacteria, PPMs that are able to cause an identifiable inflammatory response in moderately ill COPD patients.

\section{SUPPORT STATEMENT}

This study was funded by Fondo de Investigación Sanitaria PI060684, Red Respira Instituto de Salud Carlos III (ISCIII)-RTIC-03/11, and Fundació Catalana de Pneumologia. CB06/06/1089. El CIBER de Enfermedades Respiratorias (CIBERES) is an initiative of ISCIII.

\section{STATEMENT OF INTEREST}

None declared.

\section{ACKNOWLEDGEMENTS}

M.E. Kerans (Translation Office, Barcelona, Spain) reviewed the English usage in the manuscript.

\section{REFERENCES}

1 Rosell A, Monso E, Soler N, et al. Microbiologic determinants of exacerbation in chronic obstructive pulmonary disease. Arch Intern Med 2005; 165: 891-897.

2 Wilkinson TM, Hurst JR, Perera WR, et al. Effect of interactions between lower airway bacterial and rhinoviral infection in exacerbations of COPD. Chest 2006; 129: 317-324.

3 Wilkinson T, Patel I, Wilks M, et al. Airway bacterial load and FEV1 decline in patients with chronic obstructive pulmonary disease. Am J Respir Crit Care Med 2003; 167: 1090-1095.

4 Hill A, Campbell EJ, Hill SL, et al. Association between airway bacterial load and markers of airway inflammation in patients with stable chronic bronchitis. Am J Med 2000; 109: 288-295.

5 Sethi S, Evans N, Grant B, Murphy TF. New strains of bacteria and exacerbations of chronic obstructive pulmonary disease. New Engl J Med 2002; 347: 465-471.

6 Fujimoto K, Yasuo M, Urushibata K, et al. Airway inflammation during stable and acutely exacerbated chronic obstructive pulmonary disease. Eur Respir J 2005; 25: 640-646.

7 Willemse BW, TenHacken NH, Rutgers B, et al. Effect of 1-year smoking cessation on airway inflammation in COPD and asymptomatic smokers. Eur Respir J 2005; 26: 835-845.

8 Gamble E, Qiu Y, Wang D, et al. Variability of bronchial inflammation in chronic obstructive pulmonary disease: implications for study design. Eur Respir J 2006; 27: 293-299.

9 Global Initiative for Chronic Obstructive Lung Disease. Global strategy for the diagnosis, management, and prevention of chronic obstructive pulmonary disease. www.goldcopd.com Date last updated: 2007. Date last accessed: August 12, 2008.

10 Rodriguez-Roisin R. Toward a consensus definition for COPD exacerbations. Chest 2000; 117: 398s-401s.

11 Anthonisen NR, Manfreda J, Warren CPW, et al. Antibiotic therapy in exacerbations of chronic obstructive pulmonary diseases. Ann Intern Med 1987; 106: 1302-1307.

12 Burney PGJ, Chinn S. Developing a new questionnaire for measuring the prevalence and distribution of asthma. Chest 1987; 91: 79s-83s.

13 Burney P, Luczynska C, Chinn S, et al. The European Community Respiratory Health Survey. Eur Respir J 1994; 7: 954-960.

14 American Thoracic Society, Standardization of spirometry: 1987 Update. Am Rev Respir Dis 1987; 136: 1285-1298.

15 Roca J, Sanchis J, Agustí-Vidal A, et al. Spirometric reference values from a Mediterranean population. Bull Eur Physiopathol Respir 1986; 22: 217-224.

16 Pin I, Gibson PG, Kolendowicz R, et al. Use induced sputum cell counts to investigate airway inflammation in asthma. Thorax 1992; 47: 25-29.

17 Pizzichini E, Pizzichini MM, Efthimiadis A, et al. Indices of airway inflammation in induced sputum: reproducibility and validity of cell and fluid-phase measurements. Am J Respir Crit Care Med 1996; 154: 308-317.

18 Aaron SD, Angel JB, Lunau M, et al. Granulocyte inflammatory markers and airway infection during acute exacerbation of chronic obstructive pulmonary disease. Am J Respir Crit Care Med 2001; 163: 349-355.

19 Murray PR, Washington JA. Microscopic and bacteriologic analysis of expectorated sputum. Mayo Clin Proc 1975; 50: 339-344.

20 VanScoy RE. Bacterial sputum cultures. A clinician's viewpoint. Mayo Clin Proc 1977; 52: 39-41.

21 Pye A, Stockley RA, Hill SL. Simple method for quantifying viable bacterial numbers in sputum. J Clin Pathol 1995; 48: 719-724. 
22 Balows A, Hausler WJ, Herrmann KL, et al. Manual of Clinical Microbiology. 5th Edn. Washington, American Society of Microbiology, 1991.

23 Cabello H, Torres A, Celis R, et al. Bacterial colonization of distal airways in healthy subjects and chronic lung disease: a bronchoscopic study. Eur Respir J 1997; 10: 1137-1144.

24 Sethi S, Sethi R, Eschberger K, et al. Airway bacterial concentrations and exacerbations of chronic obstructive pulmonary disease. Am J Respir Crit Care Med 2007; 176: 356-361.

25 Murphy TF, Brauer AL, Sethi S, et al. Haemophilus haemolyticus: a human respiratory tract commensal to be distinguished from Haemophilus influenzae. J Infect Dis 2007; 195: 81-89.

26 Yano H, Suetake M, Kuga A, et al. Pulsed-field gel electrophoresis analysis of nasopharyngeal flora in children attending a day care center. J Clin Microbiol 1999; 38: 625-629.

27 Bhowmick A, Seemungal TAR, Sapsford RJ, et al. Comparison of spontaneous and induced sputum for investigation of airway inflammation in chronic obstructive pulmonary disease. Thorax 1998; 53: 953-956.

28 Patel I, Seemungal T, Wilks M, et al. Relationship between bacterial colonization and the frequency, character, and severity of COPD exacerbations. Thorax 2002; 57: 759-764.

29 Pillai A, Mitchell JL, Hill SL, Stockley RA. A case of Haemophilus parainfluenzae pneumonia. Thorax 2000; 55: 623-624.

30 Zervos M, Martinez FJ, Amsden GW, et al. Efficacy and safety of 3day azithromycin versus 5-day moxifloxacin for the treatment of acute bacterial exacerbation of chronic bronchitis. Int J Antimicrob Agents 2007; 29: 56-61.

31 Hoeffken G, Meyer HP, Winter J, et al. The efficacy and safety of two oral moxifloxacin regimens compared to oral clarithromycin in the treatment of community-acquired pneumonia. Respir Med 2001; 95: 553-564.

32 Middleton AM, Dowing RB, Mitrchell JL, et al. Haemophilus parainfluenzae infection of respiratory mucosa. Respir Med 2003; 97: 375-381.

33 Sethi S, Muscarella K, Evans N, et al. Airway inflammation and etiology of acute exacerbations of chronic bronchitis. Chest 2000; 118: 1557-1565.
34 Soler N, Ewig S, Torres A, et al. Airway inflammation and bronchial microbial patterns in patients with stable chronic obstructive pulmonary disease. Eur Respir J 1999; 14: 1015-1022.

35 Bresser P, Out TA, VanAlphen L, et al. Airway inflammation in nonobstructive and obstructive chronic bronchitis with chronic Haemophilus influenzae airway infection. Am J Respir Crit Care Med 2000; 162: 947-952.

36 Thanawongnuwech R, Young TF, Thacker BJ, Thacker EL. Differential production of proinflammatory cytokines: in vitro PRRSV and Mycoplasma hypopneumoniae co-infection model. Vet Immunol Immunopathol 2001; 79: 115-127.

37 Chehimi J, Trinchieri G. Interleukin-12: a bridge between innate resistance and adaptative immunity with a role on infection and acquired immunodeficiency. J Clin Immunol 1994; 14: 149-161.

38 Banerjee D, Khair OA, Honeybourne D. Impact of sputum bacteria on airway inflammation and health status in clinical stable COPD. Eur Respir J 2004; 23: 683-691.

39 Sethi S, Maloney J, Grove J, et al. Airway inflammation and bronchial bacterial colonization in chronic obstructive pulmonary disease. Am J Respir Crit Care Med 2006; 173: 991-998.

40 Papi A, Bellettato CM, Braccioni F, et al. Infections and airway inflammation in chronic obstructive pulmonary disease severe exacerbations. Am J Respir Crit Care Med 2006; 173: 1114-1121.

41 Seemungal TAR, Donaldson GC, Bhowmik A, et al. Time course and recovery of exacerbations in patients with chronic obstructive pulmonary disease. Am J Respir Crit Care Med 2000; 161: 1608-1613.

42 Chin CL, Manzel LJ, Lehman EE, et al. Haemophilus influenzae from patients with chronic obstructive pulmonary disease exacerbation induce more inflammation than colonizers. Am J Respir Crit Care Med 2005; 172: 85-91.

43 Rohde G, Wiethege A, Borg I, et al. Respiratory viruses in exacerbations of chronic obstructive pulmonary disease requiring hospitalization: a case-control study. Thorax 2003; 58: 37-42.

44 Seemugal $\mathrm{T}$, Harper-Owen R, Bhowmik A, et al. Respiratory viruses, symptoms, and inflammatory markers in acute exacerbations and stable chronic obstructive pulmonary disease. Am J Respir Crit Care Med 2001; 164: 1618-1623. 